Case Reports
in Dermatology

\title{
Retraction: “Perforating Granuloma Annulare Appearing as a Psoriasiform Lesion"
}

The article entitled "Perforating Granuloma Annulare Appearing as a Psoriasiform Lesion" [Case Rep Dermatol. 2019;11:233-8. doi 10.1159/000501875] by Benjamin M. Witkoff, Nedyalko N. Ivanov, and Shannon C. Trotter has been retracted by the Publisher and Editor.

Contrary to the submission statement signed by the authors, written informed consent from the patient for the publication of the case report was not obtained. The article is no longer available online in order to protect patient confidentiality. The authors agree to the retraction. 